\title{
Estimando o Limite de Alunos em Bate-papo Educacional: um Modelo da Participação em Bate-papo
}

\author{
Edmilson B. Rocha, Mariano Pimentel, Morganna C. Diniz \\ Departamento de Informática Aplicada - Universidade Federal do Estado do Rio de \\ Janeiro (UNIRIO) - Rio de Janeiro - RJ - Brasil \\ \{edmilson.rocha, pimentel, morganna\} @uniriotec.br
}

\begin{abstract}
Resumo. O objetivo da pesquisa apresentada é estimar quantos alunos devem participar de uma sessão bate-papo educacional de forma a obter um nível satisfatório de participação dos alunos. $O$ indicador da qualidade da participação usado é o número de mensagens enviadas por participante durante a sessão de bate-papo. Para obter essa estimativa foi elaborado um modelo matemático. Para avaliação do modelo, foram usados logs reais de bate-papos educacionais. O modelo mostrou-se válido para obter a estimativa do número de mensagens enviadas em função da quantidade de participantes.
\end{abstract}

\section{Introdução}

A Educação a Distância (EAD) no Brasil experimentou um grande crescimento a partir do ano 2000: de praticamente zero curso a distância, em uma década, esta modalidade passou a ser responsável por $15 \%$ das matrículas na graduação, totalizando cerca de um milhão de estudantes [INEP, 2012]. A mídia online já é o recurso educacional mais utilizado pelas instituições que oferecem cursos a distância [Censo EAD.BR, 2012]. Os recursos online têm ganhado cada vez mais espaço por favorecerem um novo paradigma de educação voltada para a interação. No Brasil, o bate-papo é o quarto recurso educacional mais utilizado na $\mathrm{EAD}$, ficando atrás apenas do fórum, livros e materiais impressos e do e-mail. O grande avanço da EAD e intenso uso do bate-papo nesta modalidade são o que motivaram a realização dessa pesquisa. Quando o bate-papo é usado para realizar atividades educacionais, os participantes precisam acompanhar a conversação e compreender cada mensagem. A necessidade de acompanhar a conversação é menor em bate-papo de socialização, pois os participantes não estão comprometidos em entender a conversação e até podem deixar de ler mensagens.

Alguns autores, a partir de dados empíricos e em contextos específicos, indicam que o número ideal de participantes num bate-papo é entre 4 e 5 pessoas [Böhlke 2003]; há quem defenda que o bate-papo é mais produtivo com 7 ou mais participantes [Löber et al. 2007]; há quem defenda que o grupo deve ser inferior a 10 participantes [Hrastinski 2008]. A informação divergente a respeito da quantidade de alunos que devem participar de uma sessão de bate-papo educacional se apresenta como o problema a ser equacionado nessa pesquisa. O objetivo é estimar quantos alunos devem participar de uma sessão de bate-papo educacional de forma a garantir um nível satisfatório de participação. À medida que se aumenta a quantidade de participantes numa sessão, mais mensagens são produzidas, o que demanda de cada participante mais tempo para ler todas as mensagens e assim cada indivíduo tem menos tempo para enviar novas mensagens. 


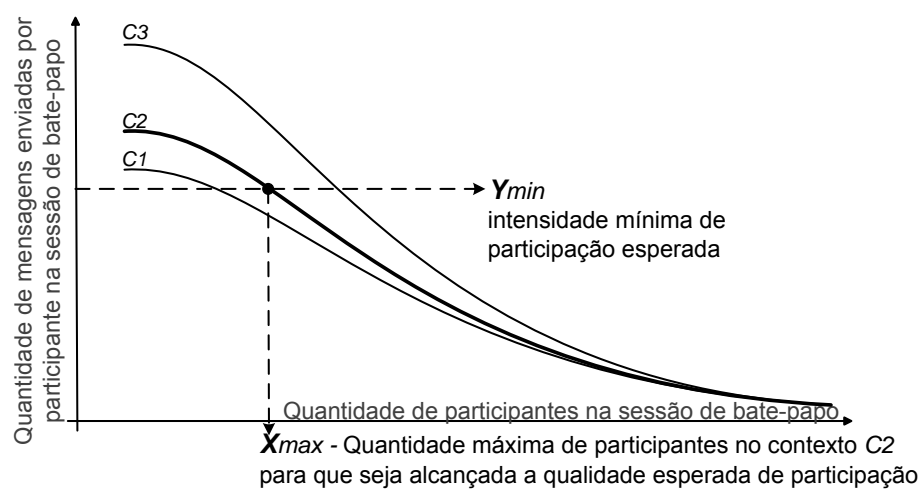

Figura 1. Modelo Idealizado de Participação em Bate-papo Educacional

Na Figura 1, é ilustrado o modelo idealizado para correlacionar a quantidade de participantes com a produção individual de mensagens na sessão. As curvas $C_{1}, C_{2}$ e $C_{3}$ representam contextos distintos (turmas diferentes), que se diferenciam por características como a idade média e nível de instrução dos participantes, assunto em discussão, dinâmica de conversação etc. Deseja-se determinar, para um dado contexto, qual a quantidade máxima de participantes $\left(X_{\max }\right)$ que pode estar na sessão para que seja possível alcançar uma determinada produção de mensagens $\left(Y_{\min }\right)$.

\section{Modelagem da participação em bate-papo educacional}

Para modelar a dinâmica de participação no bate-papo educacional foi utilizada a Teoria de Filas [Kleinrock 1975] em que cada participante foi representado como um servidor que precisa ler (atender) as mensagens (clientes) digitadas pelos participantes da sessão, enfileiradas na ordem em que são enviadas (publicadas na sala de bate-papo). Para modelar o comportamento de um participante da sessão de bate-papo educacional, foi desenvolvido um diagrama de estados para representar as atividades elementares que o participante realiza durante o bate-papo: ler e escrever mensagens - Figura 2(a).

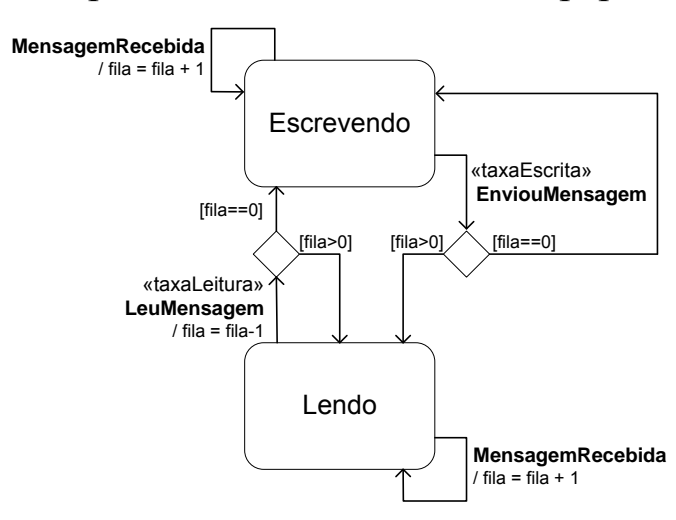

(a)

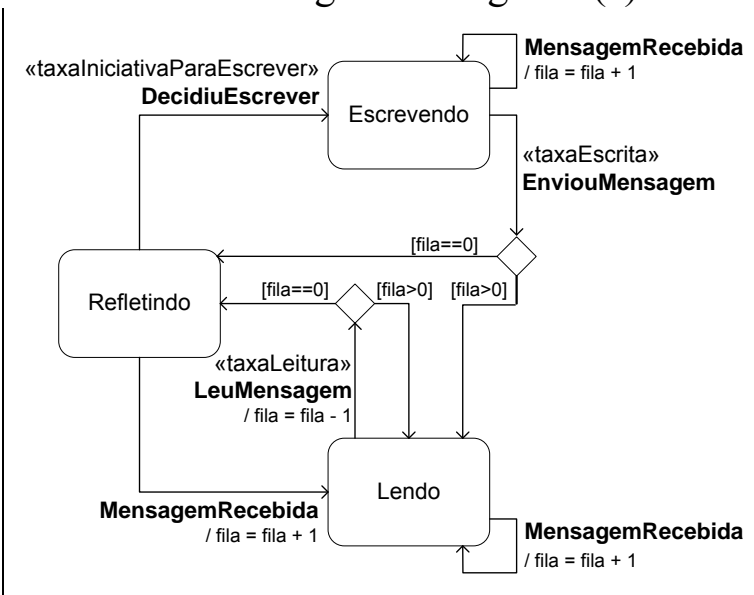

(b)

Figura 2. Diagramas de Estados do Participante

A transição de estados é modelada em função de taxas. O participante permanece Lendo uma determinada mensagem por um tempo médio de leitura $\left(\bar{T}_{L}\right)$. Da mesma forma, o participante permanece no estado Escrevendo durante um tempo médio de escrita $\left(\bar{T}_{E}\right)$. As taxaLeitura e taxaEscrita são calculadas pelo inverso do tempo médio. Representar um participante de bate-papo por apenas dois estados é irreal, pois pressupõe que o participante fica o tempo todo ou Lendo ou Escrevendo mensagens. 
Contudo, essa simplificação possibilita estimar um Limite Máximo de mensagens produzidas pelo participante. O diagrama de estados apresentado na Figura 2(b) é mais próximo do comportamento real dos usuários, pois leva em conta que um participante, além de ler e escrever mensagens, também fica Refletindo em alguns momentos.

Estando o comportamento de um participante modelado, partiu-se para a modelagem da interação entre os participantes. Numa primeira estratégia de modelagem, foi definido um objeto para representar cada participante individualmente, conforme ilustrado na Figura 3(a). Cada objeto Participante realiza as atividades apresentadas no diagrama da Figura 2(b): lê e escreve mensagens, e fica refletindo. Cada Participante também é caracterizado por suas taxas de leitura, escrita e iniciativa para escrever.

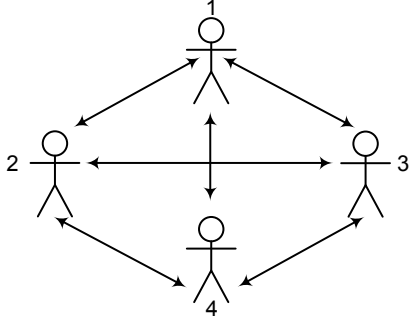

(a)

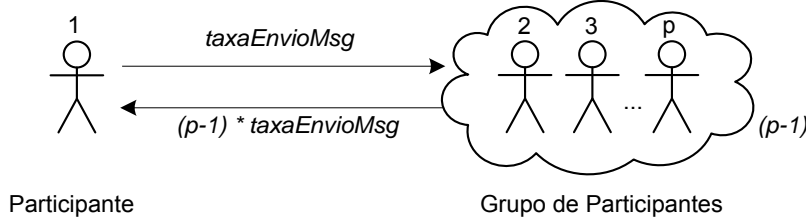

(b)

Figura 3. Estratégias de Modelagem

Nessa estratégia de modelagem, cada Participante é representado por duas variáveis: Fila e Estado. O conjunto de todos os estados dos $p$ participantes bem como as transições possíveis entre os estados são representados por uma Cadeia de Markov. Para obter as estimativas de produção de mensagem a partir dessa modelagem, o modelo foi implementado no Tangram-II [De Souza e Silva et al. 2009], que é um ambiente de modelagem baseado no paradigma orientado a objetos. Nesse contexto, o objeto Participante é replicado $n$ vezes, um para cada participante do grupo da sessão. Essa estratégia de modelagem, contudo, provoca uma explosão de estados para grupos a partir de 6 participantes. Para contornar a explosão de estados, uma segunda estratégia de modelagem foi elaborada - Figura 3(b). Nessa nova abordagem, um único objeto Participante interage com uma entidade única que representa os demais participantes do grupo produzindo mensagens. Enquanto na primeira estratégia ocorre o aumento exponencial do número de estados conforme aumenta a quantidade de participantes, na segunda estratégia apenas dois objetos interagem e o número de estados não varia em função da quantidade de participantes, logo é possível obter as estimativas de interesse.

\section{Avaliação do Modelo}

Para avaliar a adequação do modelo elaborado nesta pesquisa, as estimativas produzidas pelo modelo foram comparadas com os dados reais obtidos de logs de sessões de batepapo das turmas de uma disciplina lecionada totalmente a distância, denominada "Tecnologias de Informação Aplicadas à Educação" (TIAE) [Lucena \& Fuks 2002]. Ao todo foram obtidos $142 \operatorname{logs}$ de bate-papo de 18 turmas ministradas entre os anos de 1999 e 2008 (média por turma de 8 sessões de 1h de bate-papo). Para parametrizar o modelo por meio das três taxas que o compõe, foram usados dados obtidos dos logs das turmas TIAE em conjunto com equações formuladas por meio de estudos realizados sobre as ações elementares dos participantes de bate-papo [Rocha 2013]. 
A precisão da estimativa do modelo em relação aos dados reais é fundamentada pelo Teorema Central do Limite. Pelo Teorema, conforme aumenta o tamanho da amostra (ocorrências reais), a distribuição amostral da média aproxima-se de uma distribuição Normal, e neste caso $95 \%$ dos dados amostrais ficam entre $\mu-2 \sigma$ e $\mu+2 \sigma$, onde $\mu$ é a média populacional e $\sigma$ é o desvio padrão populacional. Optou-se por um intervalo de confiança de $95 \%$, por ser o tradicionalmente adotado em pesquisa quantitativa. Para usar o intervalo de confiança de $95 \%$, usa-se a área entre $\mu-2 \sigma$ e $\mu+2 \sigma$. Isso implica em verificar se $95 \%$ dos dados reais ocorridos nas sessões da turma TIAE ficam dentro desses limites ao redor da média estimada pelo modelo conforme Figura 4.

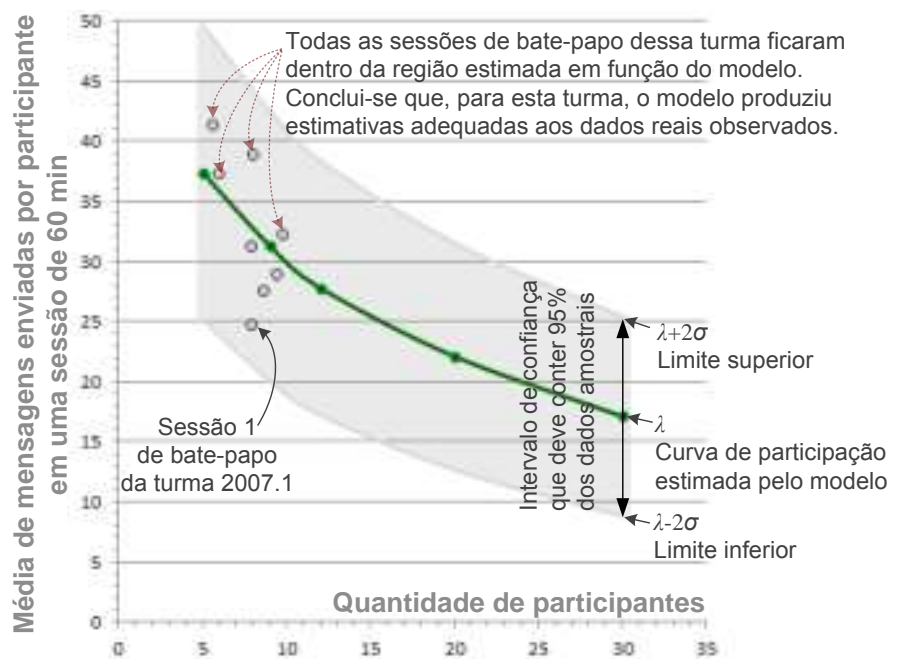

Figura 4. Dados de sessões reais de uma turma e região estimada pelo modelo

De todas as sessões de bate-papo das turmas TIAE, 95,8\% ficaram dentro da região estimada em função do modelo. Este valor é praticamente o valor exato esperado pelo Teorema Central do Limite $(95,4 \%)$, o que possibilita concluir que o modelo de participação produz estimativas adequadas. Estando validado o modelo foi possível buscar uma resposta para a pergunta inicial desta pesquisa: quantos alunos devem participar de uma sessão de bate-papo educacional? A primeira constatação sobre essa questão é que não existe uma resposta única, pois o limite de alunos depende do contexto de cada turma: do perfil dos alunos e das características da sessão de batepapo. No modelo, essas características são encapsuladas nos parâmetros Tempo Médio de Escrita, Leitura e Reflexão. Estimados esses parâmetros o modelo é usado para traçar a curva de participação de cada turma.

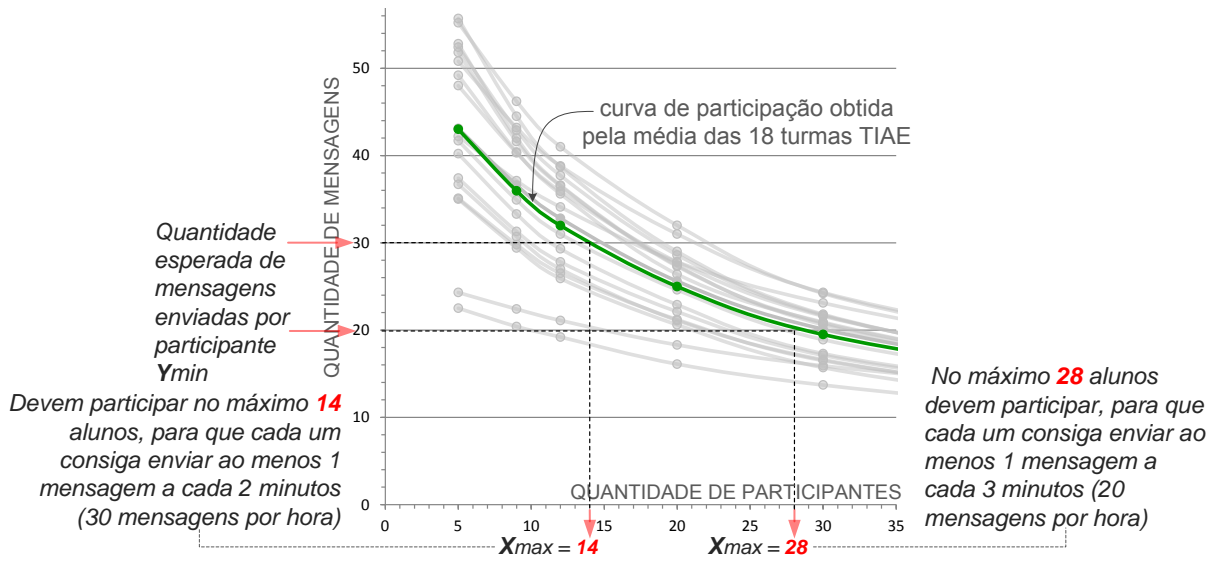

Figura 5. Quantidade de mensagens em função da quantidade de participantes 
Estudando várias turmas de uma disciplina é possível obter uma curva média para estimar a produção de mensagens em qualquer turma da disciplina. Conforme ilustrado na Figura 5, para um nível de participação de 1 mensagem a cada 3 minutos, isto é, 20 mensagens durante uma sessão de 60 minutos, pela curva identifica-se que devem participar no máximo 28 alunos. Caso o nível de participação esperado seja de 1 mensagem a cada 2 minutos (30 msg/h), deveriam participar no máximo 14 alunos.

\section{Conclusão e Trabalhos Futuros}

Os resultados produzidos pelo modelo elaborado mostraram-se adequados em comparação com os dados reais das turmas TIAE, e assim se conclui que o modelo é útil para o planejamento da quantidade de alunos que devem participar de uma sessão de bate-papo educacional de uma determinada disciplina - esse resultado é de interesse para as instituições que promovem educação online. $\mathrm{O}$ presente artigo também contribuiu para um maior entendimento sobre a participação em bate-papo educacional. Dentre as limitações dessa pesquisa estão os poucos dados obtidos dos estudos sobre os processos elementares de participação - espera-se que os processos de leitura, escrita e reflexão sejam caracterizados com mais precisão e generalização em trabalhos futuros.

\section{Referências}

Böhlke, O. (2003). A comparison of student participation levels by group size and language stages during chatroom and face-to-face discussions in German. CALICO Journal, 21 (1), 67-87.

Censo EAD.BR (2012). Organização Associação Brasileira de Educação a Distância. São Paulo: Pearson Education do Brasil $<$ http://www.abed.org.br/censoead/censoEAD.BR_2012_pt.pdf > .Acessado jun 2014

De Souza e Silva, E., Figueiredo, D., Leão, R. (2009). The TangramII integrated modeling environment for computer systems and networks. SIGMETRICS Perform. Eval. Rev., 36(4):64-69.

Hrastinski, S. (2008). The potential of synchronous communication to enhance participation in online discussions: A case study of two e-learning courses. Information \& Management, 45, 499-506.

INEP (2012). Censo da Educação Superior 2012. MEC, 2012. Disponível em < http://portal.inep.gov.br/superior-censosuperior-sinopse>. Acessado em 29 jun 2014.

Kleinrock, L., (1975). Queueing Systems - Theory, Vol. I. Wiley-Interscience. Gordon Pask, (1975), The Cybernetics of Human Learning and Performance. Hutchinson.

Löber, A., Schwabe, G., Grimm S. (2007). Audio vs. chat: The effects of group size on media choice. In Proceedings of the 40th HICCS Hawaii International Conference on System Sciences.

Lucena, C.J.P., Fuks, H. (2002). Tecnologias de Informação Aplicadas à Educação (TIAE): Manual do Aprendiz. Monografias em Ciência da Computação n.07/02, PUC-Rio.

Rocha, E. B. (2013). Modelo de Participação em Bate-papo Educacional. Dissertação de Mestrado, Universidade Federal do Estado do Rio de Janeiro - UNIRIO. 